Supporting information for

\title{
Hydrogen-assisted fast growth of large graphene grains by recrystallization of nano-grains
}

Hyunjong Lee, Jinwook Baek, Kyun Seong Dae, Seokwoo Jeon, and Jong Min Yuk*

Department of Materials Science and Engineering, Korea Advanced Institute of Science and Technology, Daejeon 305-701, Republic of Korea.

Corresponding Author*

E-mail: Jongmin.yuk@kaist.ac.kr 


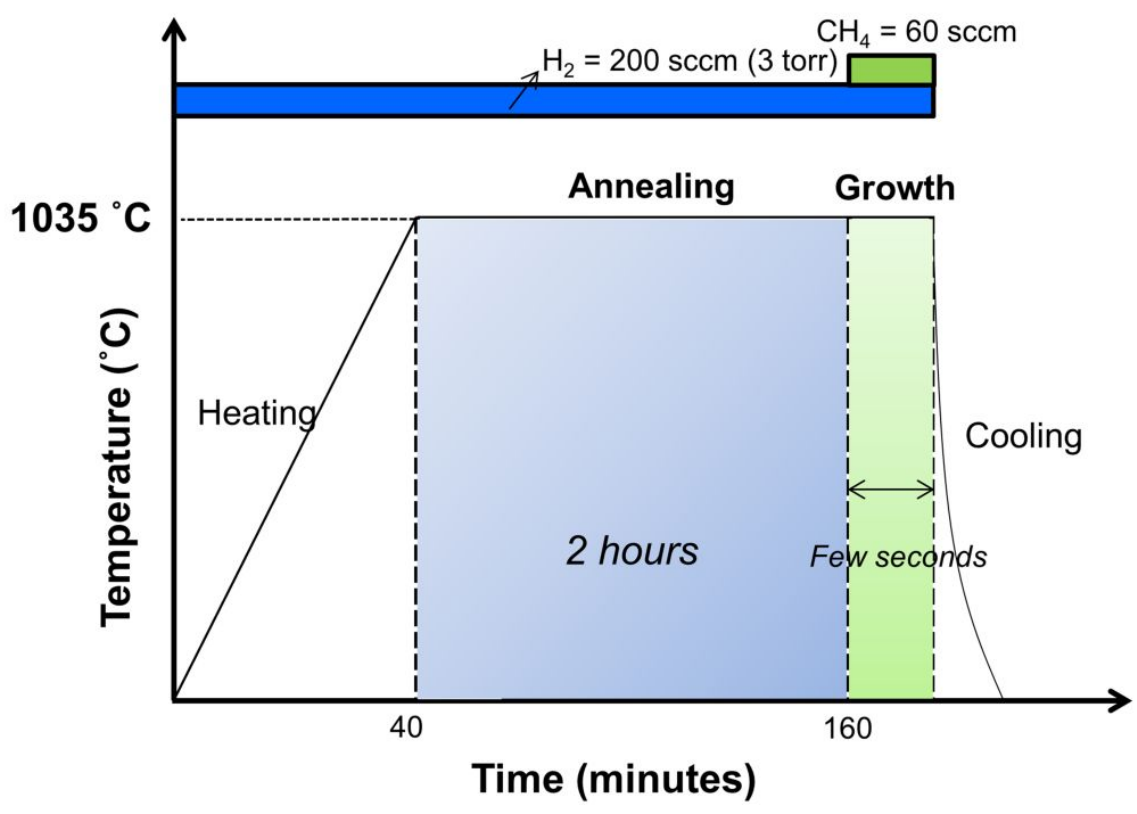

Figure S1. Schematic graph of graphene growth condition which shows fast growth of graphene with high quality. Hydrogen annealing process is proceeded at $1035{ }^{\circ} \mathrm{C}$ under $\sim 3$ torr high-pressure hydrogen $(\mathrm{HPH})$ for 2 hours and subsequent growth is conducted in a few seconds. 


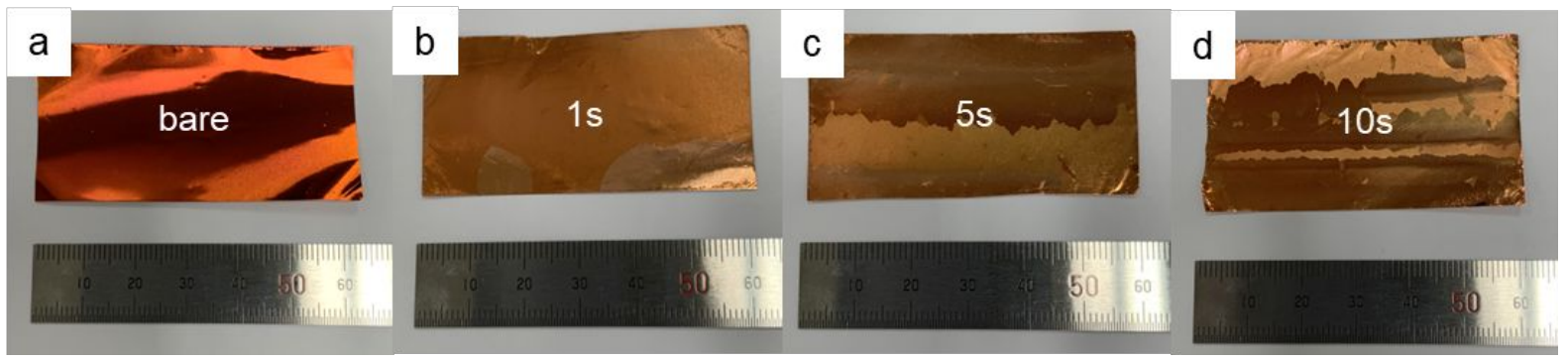

Figure S2. Photographs of $6 \mathrm{~cm} \times 3 \mathrm{~cm} \mathrm{Cu}$ foils after oxidation experiments at $200^{\circ} \mathrm{C}$ for 5 min. (a) Bare $\mathrm{Cu}$ foil, (b) a second, (c) 5 seconds, and (d) 10 seconds graphene grown $\mathrm{Cu}$ foils. Graphene-covered foils show passivation of $\mathrm{Cu}$ oxidation compared to bare foil. All foils with graphene-grown exhibit almost full coverage of $6 \mathrm{~cm} \times 3 \mathrm{~cm}$ area. 

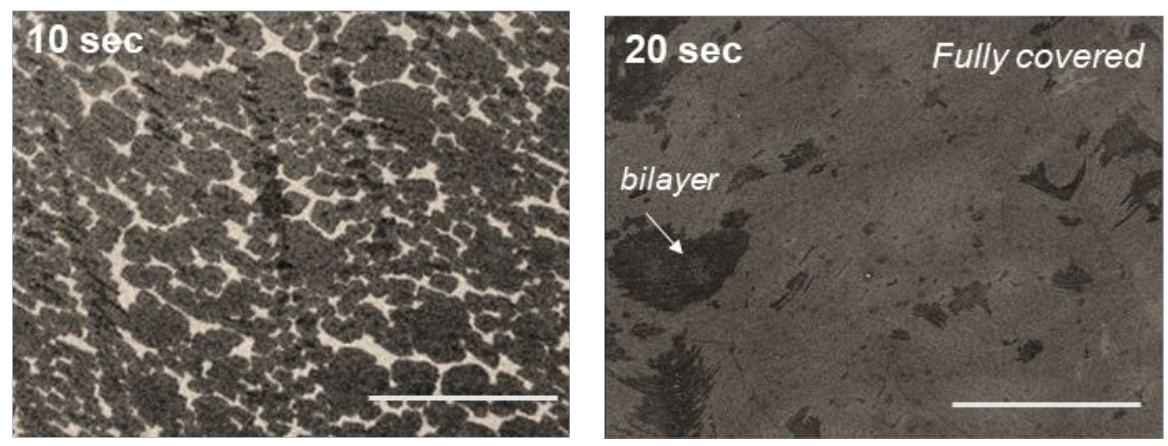

Figure S3. SEM images of graphene grown for 10 secs and 20 secs under LPH conditions. 20 secs grown specimen shows fully covered graphene on Cu foil. Scale bars indicate $10 \mu \mathrm{m}$. 

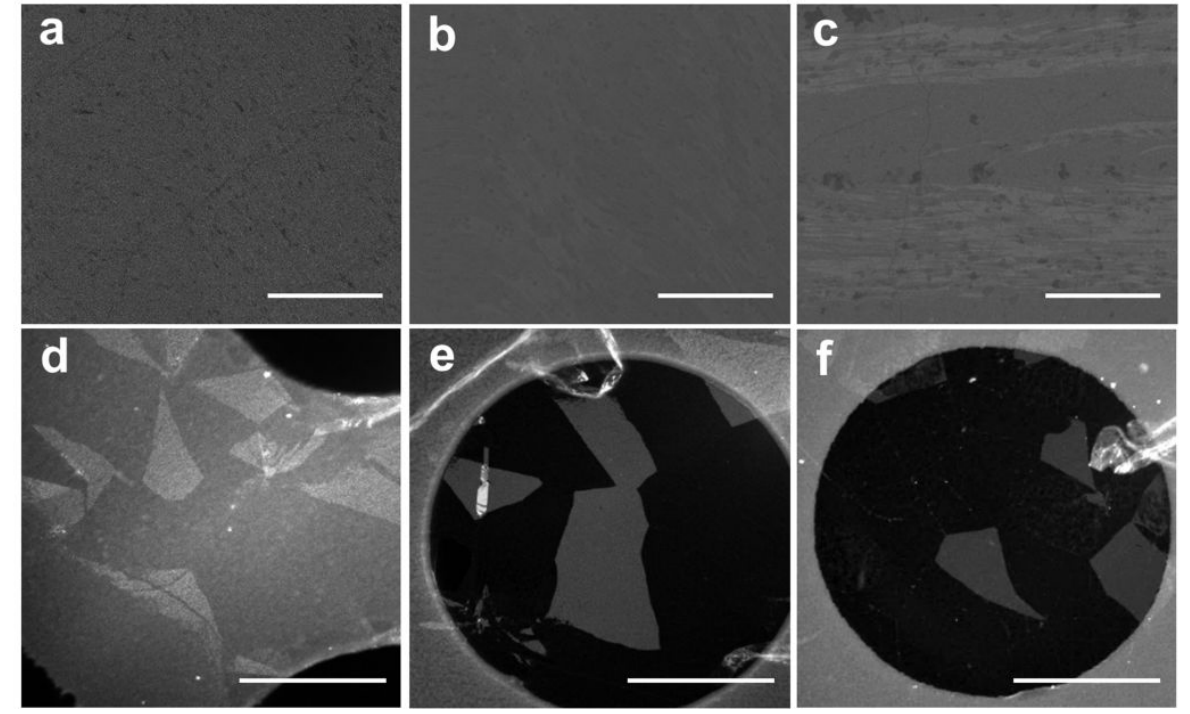

Figure S4. (a-c) SEM images of (a) 10 secs, (b) 3 min, and (c) 1 hour grown graphene on 1 torr hydrogen annealed (IPH) $\mathrm{Cu}$ foil for 2 hours. Scale bars in (a-c) indicate $10 \mu \mathrm{m}$. Dark field-TEM (DF-TEM) images of graphene grown for (d) $10 \mathrm{secs,}$ (e) $3 \mathrm{~min}$, and (f) 1 hour, respectively. Scale bars in (d-f) indicate $1 \mu \mathrm{m}$. 

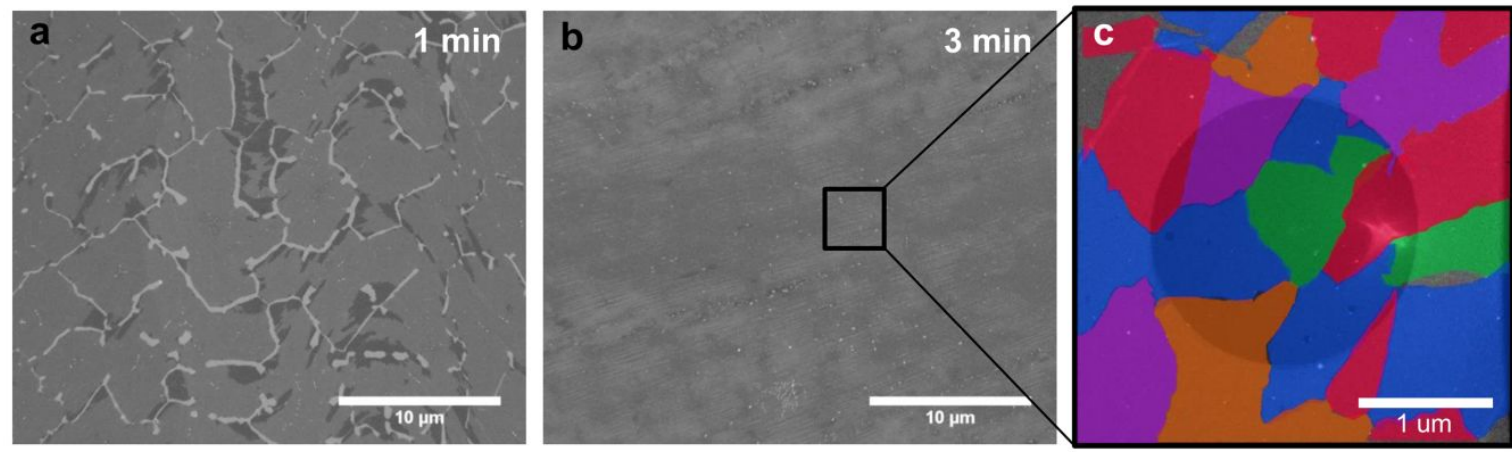

Figure S5. (a-b) SEM images of (a) 1 min and (b) 3 min grown graphene on 3 torr Ar annealed $\mathrm{Cu}$ foil. (c) False-colored DF-TEM image in 3 min grown graphene. 

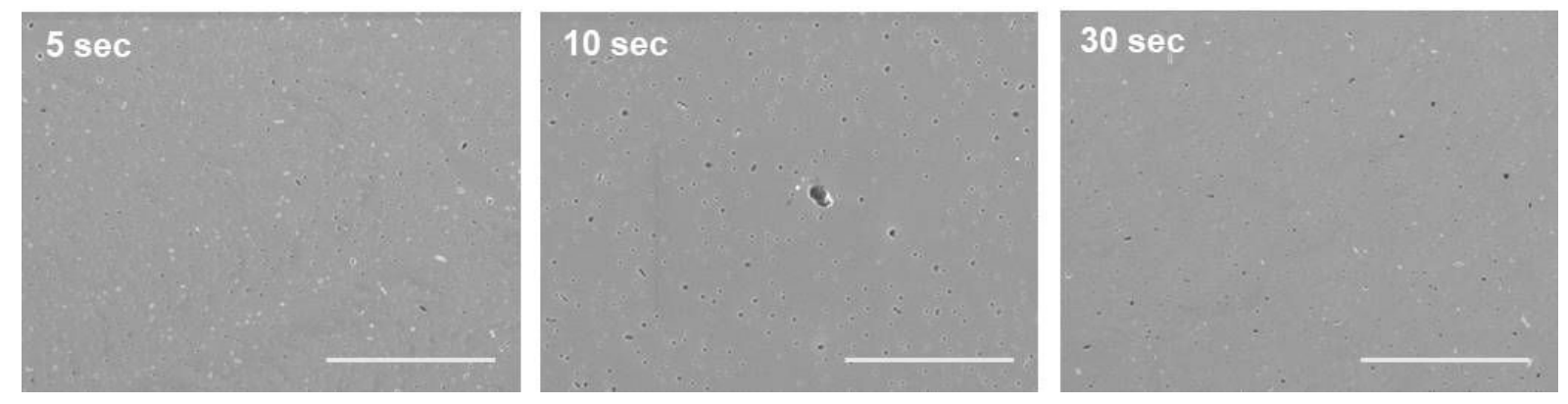

Figure S6. SEM images of graphene grown for different time under 760 torr hydrogen annealed conditions. No graphene is grown in this condition. Scale bars indicate $10 \mu \mathrm{m}$. 


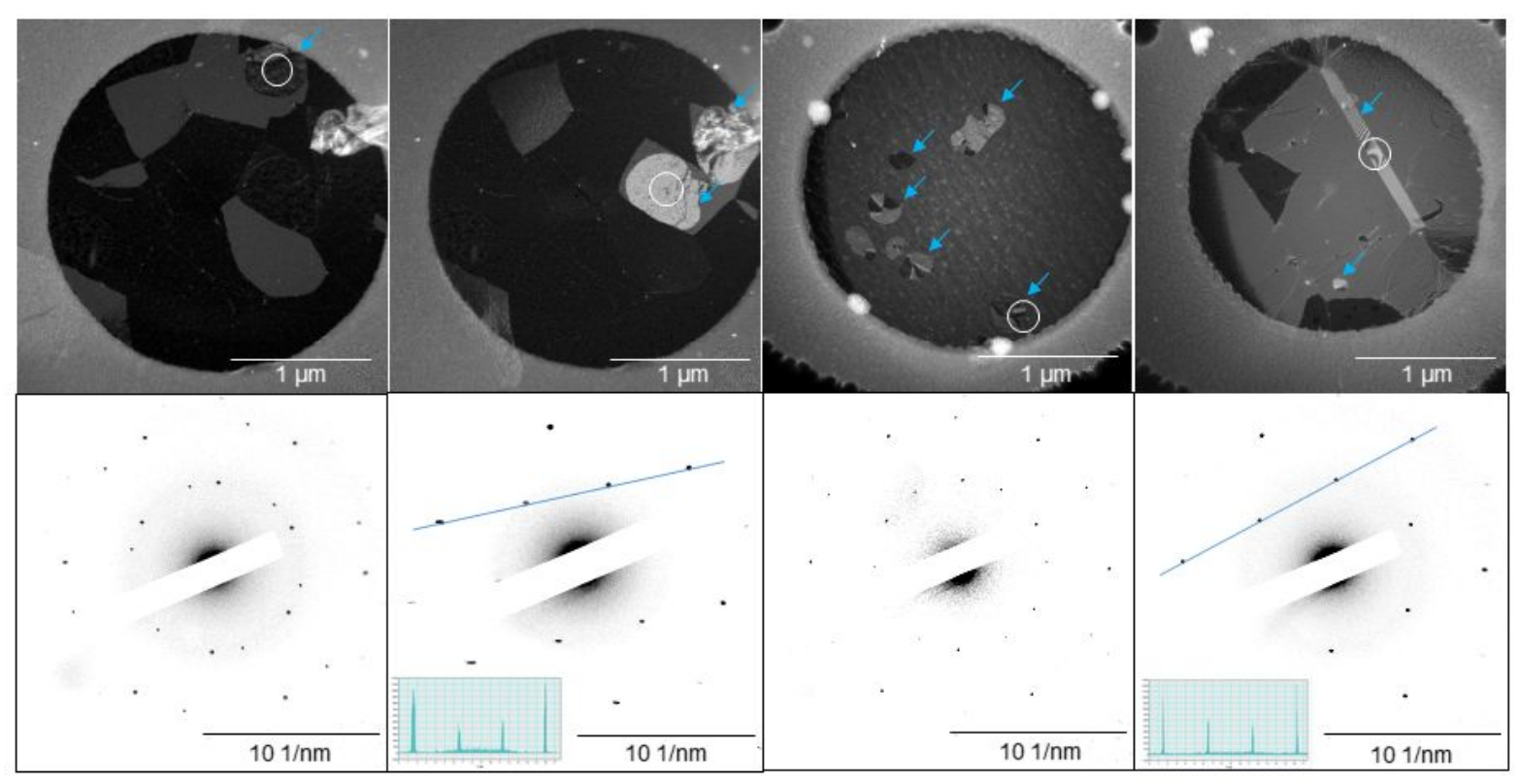

Figure S7. DF-TEM images and selected area electron diffraction patterns (SAED) of bilayer region in graphene grown for 20 secs under LPH and HPH conditions. Two images at left sides are from LPH condition and others are from HPH condition. SAED patterns of individual images are acquired at white circled regions in DF-TEM images and reveal bilayer graphene formation of twisted or bernal-stacked. Blue arrows are all indicate bilayer islands. 
a

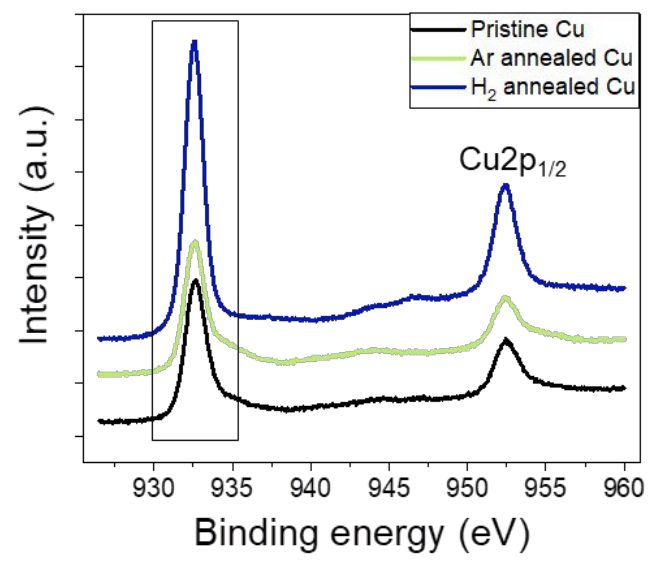

b

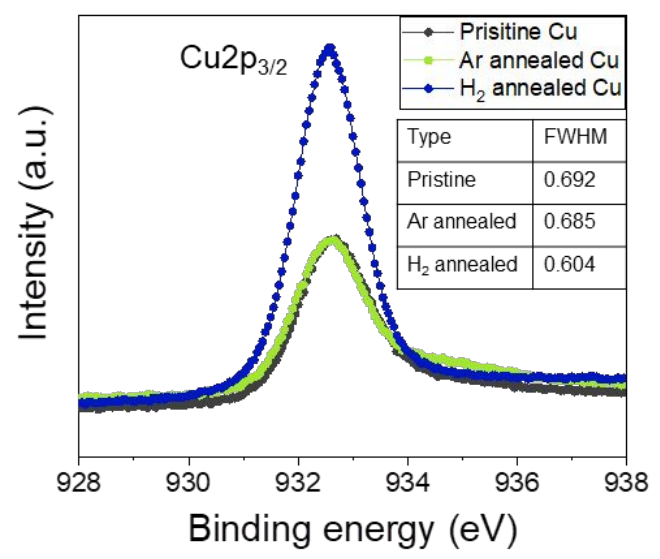

Figure S8. XPS analysis of annealed $\mathrm{Cu}$ foils at different annealing condition. (a) $\mathrm{Cu} 2 \mathrm{p}$ spectra and (b) enlarged $\mathrm{Cu} 2 \mathrm{p}_{3 / 2}$ spectra of XPS. Hydrogen annealing is conducted at HPH condition. Inset shows the full width at half maximum values at each condition. 


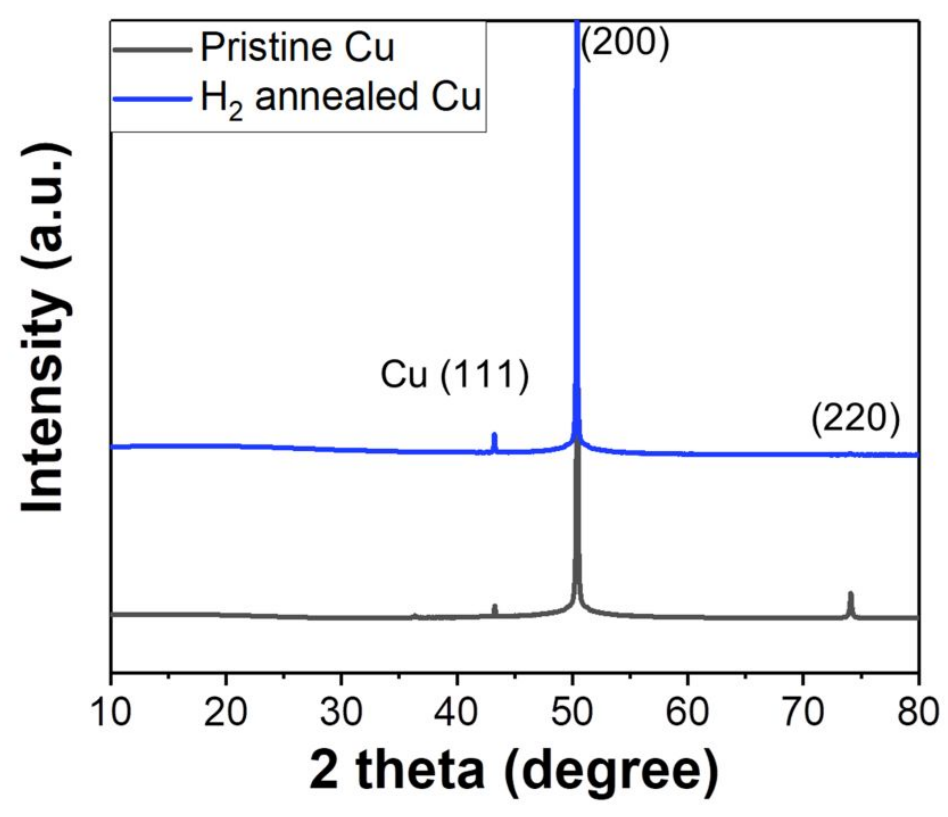

Figure S9. XRD analysis of pristine $\mathrm{Cu}$ foil and $\mathrm{HPH}$ annealed $\mathrm{Cu}$ foil. No special crystallinity difference is observed between pristine $\mathrm{Cu}$ foil and hydrogen annealed foil. 


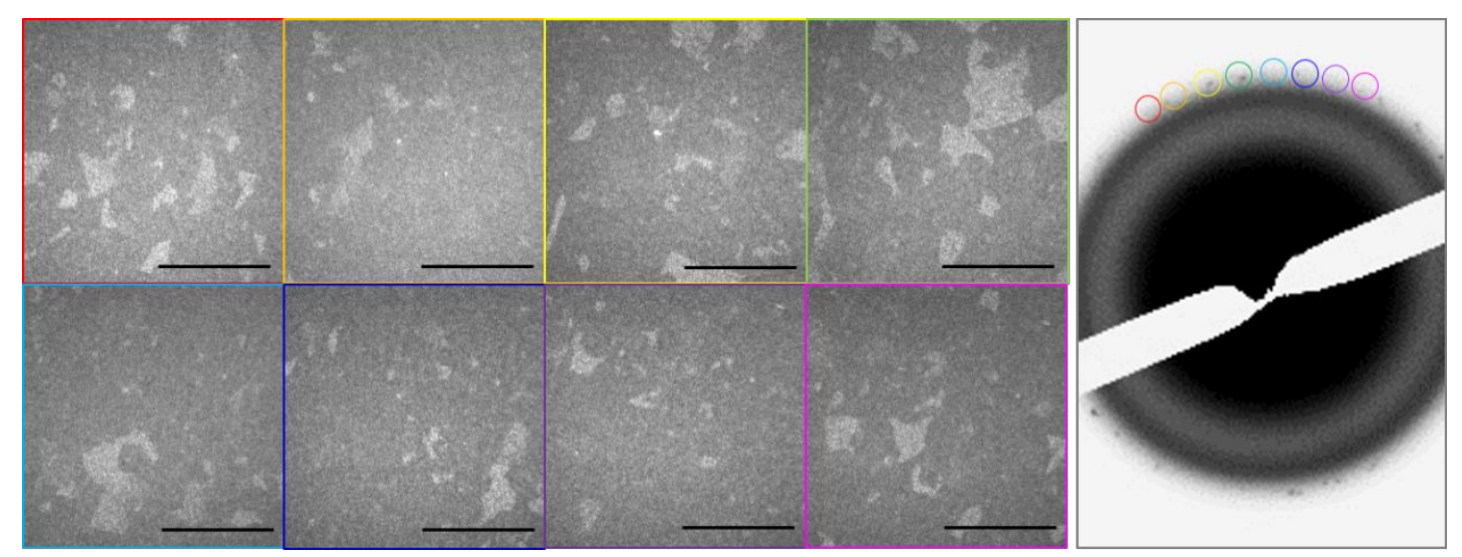

Figure S10. DF-TEM mapping images and corresponding SAED pattern of $1 \mathrm{sec}$ grown graphene at HPH condition. DF-TEM images of different color borders are acquired from diffraction spots marked by corresponding colored circles. 


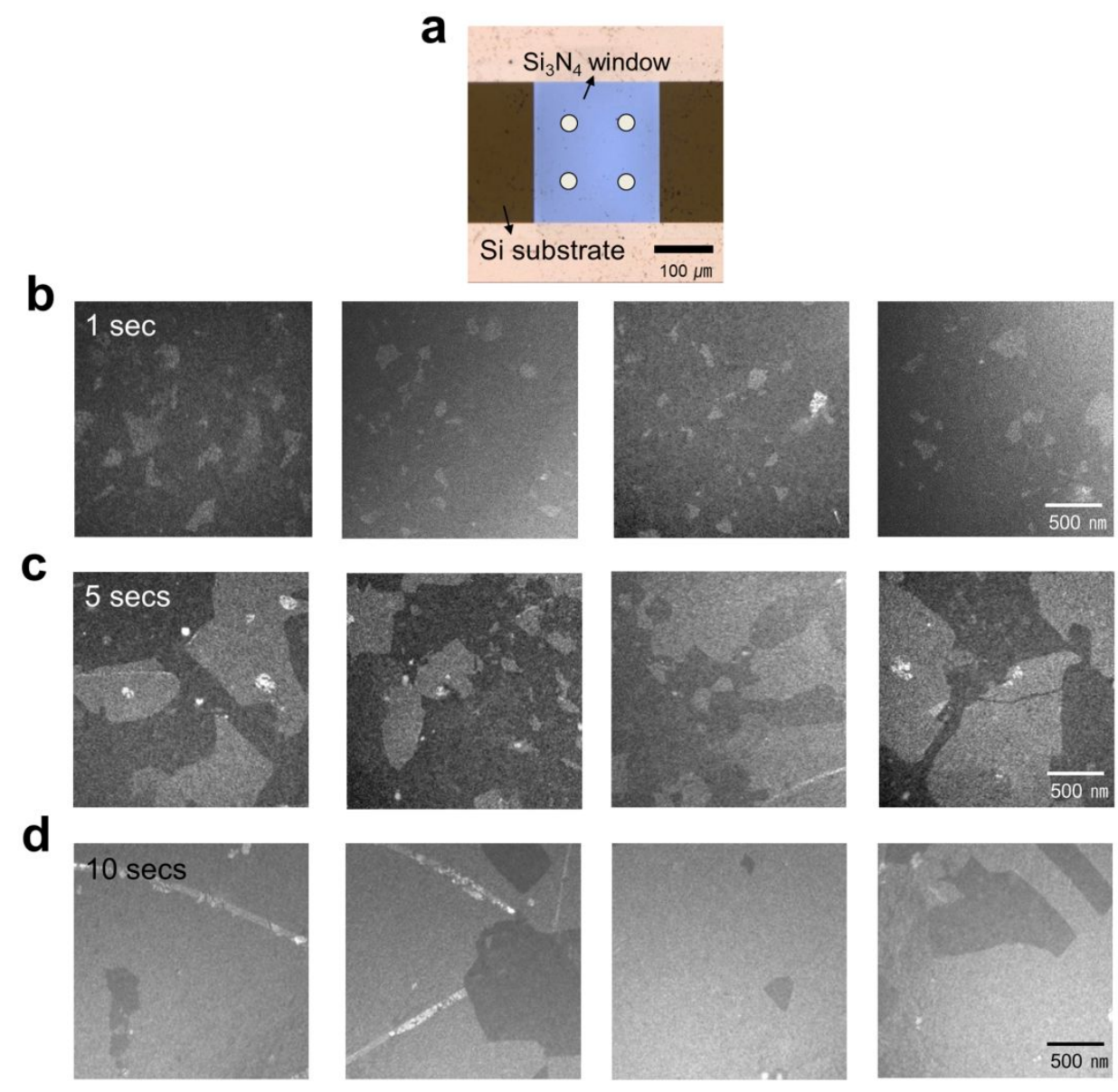

Figure S11. DF-TEM images of graphene observed at different positions of $200 \mu \mathrm{m} \times 200$ $\mu \mathrm{m} \mathrm{Si}_{3} \mathrm{~N}_{4}$ supporting film. (a) Specially designed chip for observing small grain sizes of graphene. Circles in image indicate TEM observed area. (b-d) TEM images observed in (b) a second, (c) 5 seconds, and (d) 10 seconds grown graphene. 


\begin{tabular}{|c|c|c|c|}
\hline & 1 second & 5 seconds & 10 seconds \\
\hline Maximum grain size & $\sim 950 \mathrm{~nm}$ & $\sim 21 \mu \mathrm{m}$ & $\sim 160 \mu \mathrm{m}$ \\
\hline $\begin{array}{c}\text { Ratio of grain sizes less } \\
\text { than 200nm }\end{array}$ & $\sim 90 \%$ & $\sim 65 \%$ & $\sim 25 \%$ \\
\hline
\end{tabular}

Figure S12. Maximum grain size measurements at different time grown graphene during recrystallization process. 

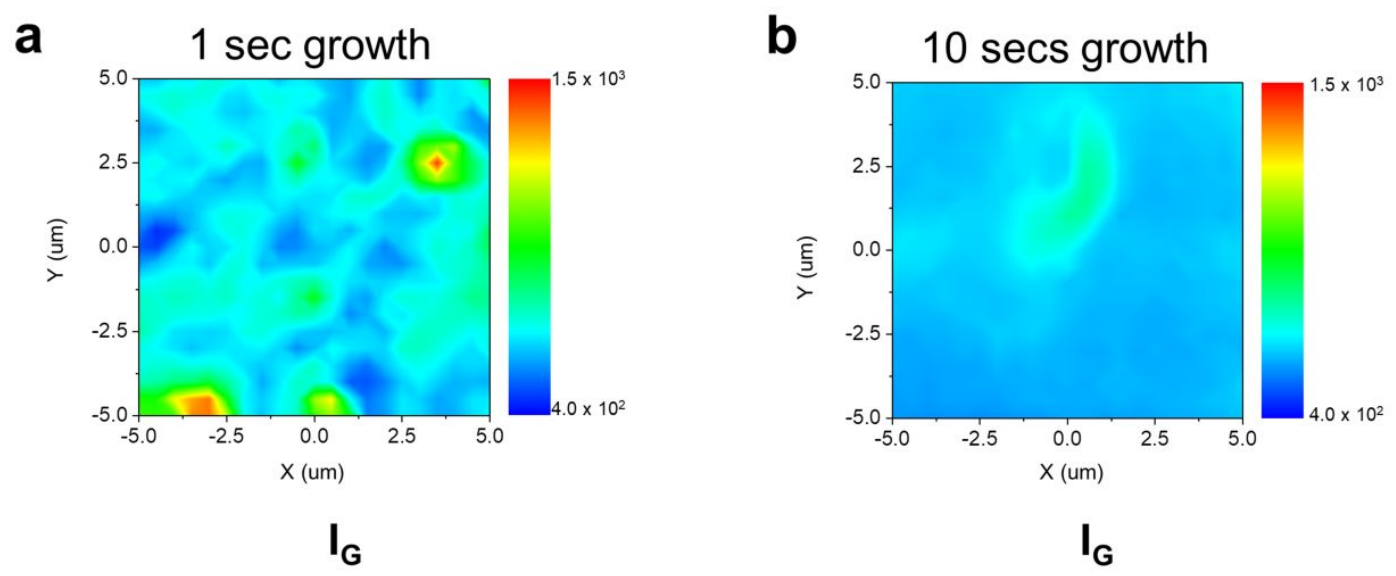

Figure S13. Two-dimensional $\mathrm{I}_{\mathrm{G}}$ Raman spectroscopy mapping of graphene grown on $\mathrm{HPH}$ annealed $\mathrm{Cu}$ foil for (a) a second and (b) 10 seconds. Laser excitation wave length is $514 \mathrm{~nm}$. 